\title{
El tratamiento intensivo con insulina reduce la mortalidad en pacientes diabéticos luego de un infarto
}

\begin{abstract}
Prospective randomised study of intensive insulin treatment on long term survival after acute myocardial infarction in patients with diabetes mellitus. Malmberg K, for the DIGAMI Study Group. BMJ 1997;314:1512-15
\end{abstract}

\section{Objetivo}

Evaluar el efecto a largo plazo del tratamiento con una infusión de insulina-glucosa seguida de un régimen intensivo de insulina luego de sufrir un infarto agudo de miocardio (IAM) en pacientes con diabetes mellitus (DM)

\section{Diseño}

Estudio randomizado, controlado. Seguimiento promedio de 3.4 años (rango 1.5-1.6).

\section{Lugar}

Unidades coronarias (UC) de 19 hospitales de Suecia.

\section{Pacientes}

Se incluyeron 620 pacientes (edad media 68 años, $63 \%$ de hombres) con un IAM $<24$ hs de evolución y glucemia $>200 \mathrm{mg} / \mathrm{dl}$. Criterios de exclusión: mal estado general; vivienda lejos de la UC o estar enrolado en otro estudio. El seguimiento fue del $100 \%$.

\section{Intervención}

Se asignó a régimen intensivo a 306 pacientes y a tratamiento usual a 314. Los pacientes asignados a régimen intensivo recibían (aparte del cuidado usual) una infusión de insulina-glucosa por 24hs y luego insulina subcutánea 4 veces/día por 3 o más meses. Los pacientes de tratamiento usual no recibían insulina excepto indicación clínica.

\section{Medición de resultados principales}

Mortalidad global anual y a largo plazo

\section{Resultados principales}

Los pacientes del régimen intensivo tuvieron menor mortalidad al año y durante todo el periodo de seguimiento (tabla). La mayor reducción de mortalidad se observó en pacientes de bajo riesgo que no recibían insulina previa al estudio.

\section{Conclusión}

La infusión de insulina-glucosa seguida de por lo menos 3 me ses de régimen de insulina intensivo redujo la mortalidad a largo plazo en pacientes con DM que habían sufrido un IAM.

Fuente de financiamiento: Hoechst Marion Roussel y Fundación Sueca Cardiopulmonar

Régimen intensivo de insulina vs. tratamiento usual

\begin{tabular}{|c|c|c|c|c|c|}
\hline Resuluato & 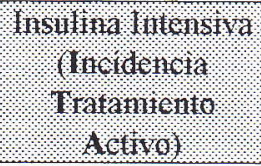 & (11) & $(1 / 4,1)$ & 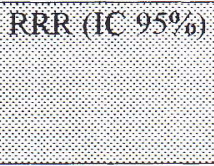 & 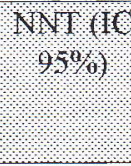 \\
\hline Mttervolat ario & $19 \%$ & $26 \%$ & $7 \%$ & $\begin{array}{c}27 \% \\
(2.5 \mathrm{a} 46 \%)\end{array}$ & $\begin{array}{c}15 \\
(7 \mathrm{a} 172)\end{array}$ \\
\hline Nuerte 10 t d nos & $33 \%$ & $44 \%$ & $11 \%$ & $\begin{array}{c}24 \% \\
(7.3 \mathrm{a} 38 \%)\end{array}$ & $\begin{array}{c}10 \\
(6 \text { a } 34)\end{array}$ \\
\hline
\end{tabular}

RRA: reducción de riesgo absoluto; RRR: reducción de riesgo relativo; NNT: número necesario para tratar

\section{Comentario}

Los pacientes diabéticos, luego de un infarto de miocardio, presentan mayor mortalidad a corto y largo plazo que los no diabéticos. Esto podría deberse a un mayor deterioro de la función ventricular izquierda; a una mayor probabilidad de reinfarto; a padecer enfermedad coronaria más extensa y más distal; a alteraciones autonómicas, de la función plaquetaria y de la fibrinolisis. Por otra parte, el tratamiento con insulina reduce las complicaciones microvasculares en diabéticos tipo I, pero no existe evidencia sobre la reducción de complicaciones macrovasculares, tanto en diabéticos tipo I como en tipo II , siendo ésta la causa más frecuente de muerte en esta población (1-2). El presente estudio se realizó para evaluar la hipótesis de que el control metabólico en pacientes diabéticos con infarto de miocardio en la fase aguda (infusión de insulina-glucosa endovenosa) (3) y en la fase crónica (insulina subcutánea) podría reducir la mortalidad en estos pacientes. La mortalidad intrahospitalaria fue menor en el grupo infusión (12,4\% vs
$15,6 \%)$, pero esta diferencia no fue estadísticamente significativa, probablemente por falta de poder para detectarla (la mortalidad observada fue menor que la esperada). Al año y a los 3,4 años, el grupo infusión presentó menor mortalidad, con una reducción de riesgo absoluto del $11 \%$, y un número necesario para tratar de 9. Las diferencias en las curvas de mortalidad se incrementaron durante todo el seguimiento. El 97\% de las causas de muerte fueron de origen cardiovascular, y muchas de ellas fueron nuevos eventos coronarios. El grupo de pacientes de bajo riesgo que no recibían insulina previa fue el más beneficiado, probablemente por el posible efecto beneficioso de la suspensión de los hipoglucemiantes orales luego del infarto. Las limitaciones metodológicas del estudio fueron: la alta proporción de pacientes excluidos (por mal estado general o dificultades en el seguimiento); un $28 \%$ de cruzamiento en el grupo infusión y $51 \%$ en el grupo control (esto podría subestimar el beneficio); la falta de ciego (aunque no hubo diferencias en- tre los grupos en relación a otras intervenciones); y el inadecuado registro del tratamiento recibido desde el alta. Este estudio no puede diferenciar si el efecto beneficioso se debe al tratamiento inicial o al tratamiento crónico. Aporta evidencia a favor del tratamiento con insulina-glucosa y luego régimen intensivo con insulina a pacientes diabéticos luego de un infarto de miocardio.

\section{Dr. Daniel Ferram: Servicio de Caralialon? Hospital Italiano de Buenoss at}

Referencias

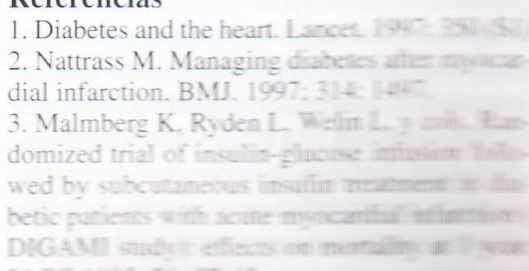

